\title{
Increasing rate of daptomycin non-susceptible strains of Staphylococcus aureus in patients with atopic dermatitis
}

\author{
Izabela Błażewicz' ${ }^{1}$ Maciej Jaśkiewicz², Lidia Piechowicz ${ }^{3}$, Damian Neubauer ${ }^{2}$, Roman J. Nowicki ${ }^{1}$, Wojciech Kamysz ${ }^{2}$, \\ Wioletta Barańska-Rybak ${ }^{1}$
}

\begin{abstract}
${ }^{1}$ Department of Dermatology, Venereology and Allergology, Medical University of Gdansk, Gdansk, Poland 2Department of Inorganic Chemistry, Faculty of Pharmacy, Medical University of Gdansk, Gdansk, Poland ${ }^{3}$ Department of Medical Microbiology, Medical University of Gdansk, Gdansk, Poland
\end{abstract}

Adv Dermatol Allergol 2017: XXXIV (6): 547-552

DOI: https://doi.org/10.5114/ada.2017.72460

\begin{abstract}
Introduction: Daptomycin is a cyclic lipopeptide that is bactericidal against Staphylococcus aureus, including methicillin-resistant S. aureus (MRSA), vancomycin-intermediate S. aureus (VISA) and vancomycin-resistant S. aureus (VRSA) strains. Daptomycin exerts its antimicrobial effect by a calcium-dependent interaction with the cytoplasmic membrane resulting in depolarization, ion loss and rapid cell death. Unfortunately, loss of daptomycin susceptibility in $S$. aureus in the clinical setting has been noted.

Aim: To evaluate the susceptibility profile to daptomycin among S. aureus strains isloted from patients with atopic dermatitis (AD). Another point was to correlate the results obtained by broth microdilution method and Etest, which is commonly applied in clinical setting.

Material and methods: One hundred patients with the diagnosis of atopic dermatitis were microbiologically assessed for the carriage of $S$. aureus. Antimicrobial susceptibility tests were performed using broth-microdilution (BMD) and Etests for daptomycin.

Results: Staphylococcus aureus strains were isolated from the majority of our patients, either from the skin (73\%) or the anterior nares (75\%). Six of the 100 nasal swabs (6\%) and 5 of the 100 skin swabs (5\%) were positive for methicillin-resistant Staphylococcus aureus (MRSA). A total of 81 of 148 (54.7\%) daptomycin non-susceptible isolates of $S$. aureus were identified by BMD. Only 19 of 81 were also classified as non-susceptible by Etest.

Conclusions: Clinicians and microbiologists should be aware of the possibility of the emergence of daptomycin non-susceptibility (or increase in minimal inhibitory concentration) during prolonged therapy and closely monitor the susceptibility of persisting isolates that might be recovered during therapy.
\end{abstract}

Key words: atopic dermatitis, broth-microdilution, daptomycin, Staphylococcus aureus, vancomycin.

\section{Introduction}

Atopic dermatitis $(A D)$ is a chronic, pruritic skin disease mainly affecting children which follows a remitting and relapsing course. It occurs in $10 \%$ to $20 \%$ of children and $1 \%$ to $3 \%$ of adults. Patients with AD have a unique predisposition to be colonized or infected by a number of microbial organisms, mostly Staphylococcus aureus. Eighty percent to $100 \%$ of patients with AD present nasal or skin colonization by $S$. aureus, while the prevalence in healthy individuals is $5 \%$ to $30 \%$. An exacerbation of AD can be associated with bacterial infection; staphylococcal infections are the most common. An attempt was made to prove that eradication of S. aureus significantly reduces the severity of the disease [1-3]. Therapeutic options for multidrug-resistant Gram-positive pathogens such as methicillin-resistant Staphylococcus aureus (MRSA) are limited. Daptomycin (DAP) seemed to be a promising candidate for new classes of anti-infectives. Daptomycin is a calcium-dependent cyclic lipopeptide produced by Streptomyces roseosporus, which shows a potent bactericidal activity against most Gram-positive organisms including MRSA [4]. Daptomycin has a clinically relevant activity against a variety of van-

Address for correspondence: Izabela Błażewicz, Department of Dermatology, Venereology and Allergology, Medical University of Gdansk, 1a Kliniczna St, 80-402 Gdansk, Poland, fax: +48 7910023 15, e-mail: izabela.blazewicz@wp.pl Received: 7.06 .2016 , accepted: 4.10.2016. 
comycin-resistant organisms including S. aureus [5]. The mechanism of daptomycin action is unique: the drug kills bacteria in a concentration-dependent manner by binding preferential membranes of Gram-positive bacteria. After insertion, rapid depolarization occurs which leads to death of the bacterial cells due to disruption of critical metabolic functions, such as protein, DNA, and RNA synthesis [6]. Owing to its unique to its unique mechanism of action, it has been generally assumed that daptomycin-resistant organisms are difficult to generate. Unfortunately, therapeutic failures, albeit relatively uncommon, have been reported $[7,8]$. Such non-susceptibility may occur in the absence of prior daptomycin exposure.

\section{Aim}

The main purpose of this study was to determine the antimicrobial susceptibility to daptomycin of $S$. aureus strains among patients with atopic dermatitis. In the present study, we also evaluated the correlation of daptomycin minimal inhibitory concentration (MIC) obtained by the Etest technique and the broth microdilution (BMD) method.

\section{Material and methods}

\section{Patients and bacterial strains}

Patients were enrolled in our study at the time of their visits to the Outpatient Clinic and during hospitalization in the Department of Dermatology, Venereology and Allergology in Gdańsk from August 2014 to August 2015. There was no selection of patients by sex or by severity of lesions. Atopic dermatitis (AD) was diagnosed following the criteria of Hanifin and Rajka, which include pruritus, typical morphology and distribution of eczematous lesions, chronicity of the disease and personal or family history of atopy [9].

The study was approved by the local Research Ethics Board (approval number NKBBN/242-477/2014). Voluntary informed consent in writing was obtained from all participants. The exclusion criteria included chronic dermatological condition with compromised skin barrier (e.g. psoriasis), diagnosis of any other chronic condition that increases the risk of MRSA colonization, oral or intravenous antibiotic treatment in the previous 4 weeks, treatment with topical antibiotics in the past 2 weeks, treatment with systemic corticosteroids or immunosuppressive drugs in the past 4 weeks, history of hospitalization, surgery, dialysis or residence in a long-term facility in the past year, indwelling catheter or a percutaneous device at the time of enrollment. Skin and nasal swabs were collected from 100 patients with AD to investigate the presence of $S$. aureus. The definition of Community Acquired-MRSA (CA-MRSA) was coined by the Centers for
Disease Control and Prevention (CDC) in 2000. It refers to MRSA infection in a person who has none of the following established risk factors: isolation of MRSA more than $48 \mathrm{~h}$ after hospital admission; history of hospitalization, surgery, dialysis or residence in a long-term care facility within one year of the MRSA culture date; the presence of an indwelling catheter or a percutaneous device at the time of culture; or previous isolation of MRSA.

\section{Identification of $S$. aureus and MRSA strains}

Preliminary identification and detection of S. aureus and MRSA strains was conducted on the ChromID MRSA/ ChromID S. aureus biplate (bioMérieux) for the simultaneous detection of $S$. aureus and MRSA.

\section{Antimicrobial activity}

All S. aureus strains were used to determine the MIC using the broth microdilution method in Mueller Hinton broth according to the Clinical and Laboratory Standards Institute (CLSI) recommendations [10]. Assays for daptomycin were performed with and without medium supplemented with $\mathrm{Ca}^{2+}(50 \mathrm{mg} / \mathrm{l})$. Polypropylene 96-well plates with bacteria at initial inoculums of $0.5 \times 10^{5} \mathrm{CFU} /$ $\mathrm{ml}$ were exposed to daptomycin ranging concentrations $(0.0625-32 \mu \mathrm{g} / \mathrm{ml})$. All plates were further incubated for $18 \mathrm{~h}$ at $37^{\circ} \mathrm{C}$. Minimal inhibitory concentration was taken as the lowest concentration of the compound at which a visible growth of bacteria was not observed. The experiments were performed in triplicate. Daptomycin non-susceptible strains (MIC >1 $\mathrm{\mu g} / \mathrm{ml}$ ) were selected for Etests (bioMérieux). Mueller-Hinton (BBL) agar plates were used for the Etest. All setup and reading procedures were based on the manufacturer's instructions [11].

\section{Statistical analysis}

All statistical calculations were performed using the statistical package StatSoft. Inc. (2014) Statistica (data analysis software system) version 12.0. www.statsoft. com and Excel spreadsheet. Quantitative variables were characterized by the arithmetic mean, standard deviation, median, minimum and maximum values (range) and $95 \% \mathrm{Cl}$ (confidence interval).

To check whether a variable quantitative came from a normally distributed population, the Shapiro-Wilk analysis was used. In contrast, to test the hypothesis of equal variances, Leven (Brown-Forsythe) test was used.

The significance of differences between two examined groups, Student's $t$ test or Mann-Whitney $U$ were used. The significance of differences between more than two groups were checked by an F test (ANOVA) or Kruskal-Wallis. In all the calculations, the level of significance was assumed at $p=0.05$. 
Table 1. Susceptibility profile to Daptomycin of tested Staphylococcus aureus strains isolated from patients with atopic dermatitis (AD). Minimal inhibitory concentration was determined using the broth-microdilution method (BMD) with and without $\mathrm{Ca}^{2+}(50 \mathrm{mg} /)$ supplementation (148 strains). Daptomycin non-susceptible strains (MIC $>1 \mu \mathrm{g} / \mathrm{ml}$ ) were selected for Etest confirmation (81 strains)

\begin{tabular}{lccc}
\hline No. of isolates & \multicolumn{3}{c}{ MIC range $[\mu \mathrm{g} / \mathrm{ml}]$} \\
\cline { 2 - 4 } & BMD & BMD $\left(\mathrm{Ca}^{2+}\right)$ & Etest \\
\hline $\begin{array}{l}\text { S. aureus isolated from patients with AD } \\
(148)\end{array}$ & $0.25-16$ & & \\
\hline $\begin{array}{l}\text { S. aureus Daptomycin non-susceptible } \\
(81)\end{array}$ & $8.0-16$ & $2.0-4.0$ & $\mathrm{n} / \mathrm{a}$ \\
\hline
\end{tabular}

n/a-not applicable.

Table 2. MIC50 and MIC90 values to Daptomycin of tested Staphylococcus aureus strains isolated from patients with AD. Minimal inhibitory concentration was determined using the broth-microdilution method (BMD) with and without $\mathrm{Ca}^{2+}(50 \mathrm{mg} / \mathrm{l})$ supplementation (148 strains). Daptomycin non-susceptible strains ( $\mathrm{MIC}>1 \mu \mathrm{g} / \mathrm{ml}$ ) were selected for Etest confirmation (81 strains)

\begin{tabular}{|c|c|c|c|c|c|c|}
\hline \multirow[t]{3}{*}{ No. of isolates } & \multicolumn{6}{|c|}{$\mathrm{MIC}$ range $[\mu \mathrm{g} / \mathrm{ml}]$} \\
\hline & \multicolumn{2}{|c|}{ BMD } & \multicolumn{2}{|c|}{$\mathrm{BMD}\left(\mathrm{Ca}^{2+}\right)$} & \multicolumn{2}{|c|}{ Etest } \\
\hline & MIC50 & MIC90 & MIC50 & MIC90 & MIC50 & MIC90 \\
\hline $\begin{array}{l}\text { S. aureus isolated from patients with } A D \\
(148)\end{array}$ & 8.0 & 16 & 2.0 & 4.0 & $\mathrm{n} / \mathrm{a}$ & $\mathrm{n} / \mathrm{a}$ \\
\hline $\begin{array}{l}\text { S. aureus Daptomycin non-susceptible } \\
\text { (81) }\end{array}$ & 8.0 & 16 & 2.0 & 4.0 & 0.25 & 16 \\
\hline
\end{tabular}

n/a-not applicable.

\section{Results}

\section{Patients and bacterial strains}

A total of 200 specimens were collected from 100 patients during the study. AD patients consisted of $55 \%$ of males and 45\% of females, age: 1 to 63 years, median: $22.3 \pm 15.6$ years. Staphylococcus aureus was reported in 75 of $100(75 \%)$ skin swabs and 73 of $100(73 \%)$ nasal swabs. Six of the 100 nasal swabs (6\%) and 5 of 100 skin swabs (5\%) were positive for MRSA (54.5\% Community Acquired-MRSA, 45.5\% Hospital Acquired-MRSA).

\section{Antimicrobial susceptibility}

In the following study, none of $S$. aureus strains were characterized as resistant to vancomycin in both, BMD method and Etest. In case of daptomycin, MIC determination was followed by two different methods - a broth microdilution (BMD) method and a gradient diffusion strip method (Etest). BMD was conducted in reference to the Clinical and Laboratory Standards Institute recommendations. Interestingly, the influence of $\mathrm{Ca}^{2+}$ medium supplementation $(50 \mathrm{mg} / \mathrm{l})$ was also examined. The Etest method was conducted according to manufacturer's instructions. In the first step, a total of 81 of 148 (54.72\%) non-daptomycin-susceptible strains of $S$. aureus were identified by BMD in patients with AD. Strains isolated from patients with AD, which were non-susceptible to daptomycin were selected to
Etest. Minimal inhibitory concentration determined in $\mathrm{Ca}^{2+}$ supplemented medium was 2-fold dilution lower (97.50\% of strains) than in standard Mueller-Hinton medium (Table 1). For Etest, only 19 of 81 (23.45\%) nonsusceptible strains were classified as resistant. Minimal inhibitory concentration values generated with this method tended to be lower. In majority, up to 3 and 4 dilutions but +7 and -7 variations were also noted. There was also a poor correlation between BMD and Etest (correlation coefficient, $r=0.306$ for confidence interval 0.95$)$. Only $14.81 \%$ of the MIC values were within 1 dilution for values obtained by BMD. Minimal inhibitory concentration 50 and MIC 90 values for both methods were diametrically different (Table 2). For BMD it was $2.0 \mu \mathrm{g} / \mathrm{ml}$ and $4.0 \mu \mathrm{g} / \mathrm{ml}$, respectively, while for Etest it was $0.25 \mu \mathrm{g} / \mathrm{ml}$ and $16 \mu \mathrm{g} / \mathrm{ml}$. Since there is no intermediate interpretive category for daptomycin, only very major or major category interpretive errors can occur. In contrast to the BMD method, there were $76.54 \%$ of major errors (false susceptibility) determined by Etest.

\section{Discussion}

Epidemiology of Staphyloccocus aureus and MRSA strains in atopic dermatitis

Staphylococcus aureus strains were isolated from the majority of our patients, either from the skin (75\%) or the anterior nares (73\%). These results suggest that 
the nose may act as a reservoir of S. aureus transferred from the skin surface by autotransmission. In 1961, first MRSA strains were reported. In 1980, MRSA strains became an endemic problem at different proportions at hospitals in several countries. Traditionally, infections caused by MRSA were limited to hospitals (Hospital Acquired-MRSA, HA-MRSA). Community-acquired infections (Community Acquired-MRSA, CA-MRSA) have been increasingly recorded since the last decade. The first report on CA-MRSA infection in a patient without any contact with the hospital environment was recorded in 1980 in the United States. There are few epidemiological reports on the colonization of methicillin-resistant S. aureus in atopic dermatitis. Worldwide studies suggest that the prevalence of MRSA in the population with AD varies from 0 to $30.8 \%$ [12]. In the USA, where CA-MRSA is now the most common pathogen cultured from patients with skin and soft-tissue infections in emergency departments, the colonization rate of AD patients is as high as $18.3 \%$ [13]. In the presented study, 6 of the 100 nasal swabs (6\%) and 5 of 100 skin swabs (5\%) were positive for MRSA (54.5\% C-MRSA, 45.5\% H-MRSA).

\section{Daptomycin resistance}

Daptomycin (DAP) is the first member of the antimicrobial agents approved for clinical use. Advantageous characteristics include a long serum half-life allowing once-daily dosing as well as antimicrobial activity against bacteria with reduced glycopeptide susceptibility [5].

Daptomycin is nowadays considered to be an antibiotic of choice for the treatment of biofilm-related infections. Daptomycin has been investigated with in vitro biofilm models $[14,15]$. The rapid effectiveness is supported further by the observation that daptomycin eradicated MRSA in a biofilm after 3 days of 4-h daily exposures [16]. It was shown to act faster than minocycline, tigecycline, linezolid, vancomycin, and rifampin against in vitro central venous catheter biofilm infections [16].

The range of potential adaptations that may be associated with staphylococcal DAP resistance includes increased positive surface charge ('charge-repulsion hypothesis'), altered cell membrane fatty acid composition resulting in altered fluidity ('membrane order hypothesis'), enhanced cell membrane content of positivelycharged phospholipids, as well as increased D-alanylation of the cell wall teichoic acid, resulting in reduced affinity of DAP to the cell membrane target, reduced permeabilization capacities, and reduced depolarization [17]. Mutations in mprF (a gene which contributes to membrane charge through lysinylation of $P G$ ), yycG (a histidine kinase gene of multiple functions, including impacts on membrane fatty acid biosynthesis), and rpoB and rpoC (subunits of RNA polymerase) have been found in S. aureus strains with daptomycin MIC greater than the susceptible range [18].
Several reports have linked increases in vancomycin MIC to increases in daptomycin MIC although no definitive mechanism has been elucidated. It may include increased cell wall thickness or reduced autolysis phenotypes, as commonly exhibited by strains with reduced susceptibility to vancomycin $[19,20]$. However, such an association has not been seen in the presented study. The observation mentioned above suggests that frequent practice of using daptomycin when vancomycin therapy appears to be failing may be the wrong strategy.

Recent data have shown "cross-resistance" between DAP and cationic host defense peptides (HDPs) from neutrophils and platelets in S. aureus strains obtained from patients failing DAP therapy. Similar to many endogenous HDPs, daptomycin contains a significant peptide moiety that can be positively charged by calcium decoration during in vivo use. Therefore, one potential driver of such HDP-daptomycin cross-resistance phenotypes may be the capacity of innate HDPs to impact organisms before daptomycin therapy, facilitating increased daptomycin MICs on subsequent daptomycin exposure [21, 22]. Since many of the strains in the presented study were isolated from skin infection, it is quite possible to exhibit the daptomycin nonsusceptibility during therapy because daptomycin exhibits cross resistance to other cationic host defense peptides.

\section{Susceptibility testing}

Susceptibility breakpoints for daptomycin are currently $\leq 1 \mu \mathrm{g} / \mathrm{ml}$ for methicillin-susceptible S. aureus (MSSA) and MRSA strains. Criteria for intermediate susceptibility or resistance have not been established because of lack of such strains. Organisms with a daptomycin MIC of $>1 \mu \mathrm{g} / \mathrm{ml}$ are considered non-susceptible. The Clinical and Laboratory Standards Institute (CLSI) recommends confirmation of non-susceptible strains by another method [11]. The European Committee on Antimicrobial Susceptibility Testing (EUCAST) has set both susceptible (MIC $\leq 1 \mathrm{mg}$ / $\mathrm{ml}$ ) and resistant (> $1 \mathrm{mg} / \mathrm{ml}$ ) breakpoints for Staphylococcus. Disk diffusion testing is not recommended for daptomycin by the CLSI, EUCAST, or FDA until the method can be adjusted for appropriate detection of daptomycinnonsusceptible strains. In contrast, Etest is frequently used by clinical laboratories to determine daptomycin MIC. The presented study confirms the previous reports that the concentration-dependent bactericidal activity of daptomycin requires physiological levels of free calcium ions $(50 \mathrm{mg} / \mathrm{ml})$ [23]. In the following study MIC determined in $\mathrm{Ca}^{2+}$ supplemented medium was 2-fold dilution lower (97.50\% strains) than in standard Mueller-Hinton medium. Studies with daptomycin Etest by Fuchs et al. documented the pronounced effect of various calcium concentrations on MIC results [24]. Studies comparing the MIC results between the revised Etest method and the results of broth microdilution testing staphylococci have been reported recently [25]. Previous studies demonstrating a poor correlation among different methods for determining suscep- 
tibility against daptomycin have reported that the Etest shows higher MIC results than the BMD reference method [26]. The lack of an intermediate category means that any category errors between a test method and the reference susceptibility test method can be categorized as either major (false resistance) or VM (false susceptibility). Further studies revealed 13\% to 100\% very major errors (VME) among MRSA strains with daptomycin-non-susceptibility MIC by BMD [27]. This study clearly demonstrates that there is a poor correlation of MIC results among the different methods used for susceptibility testing of daptomycin. Clinicians should be aware of the difficulties associated with susceptibility testing of daptomycin and interpret the non-susceptible results with caution.

\section{Conclusions}

Staphylococcus aureus presents significant clinical challenges because of its rising prevalence of antimicrobial resistance. Methicillin-resistant S. aureus (MRSA) infections have become a general occurrence in hospitals, and the situation is worrying since the pathogen is resistant to many antibiotics, including daptomycin and vancomycin, which were considered as the last resort for treatment of MRSA infections. Unfortunately, therapeutic failures have been reported, which correspond well with our findings (54.7\% of strains non-susceptible to daptomycin). The possible explanation of a high number of the above-mentioned strains may be cross-resistance between daptomycin and cationic host defense peptides from neutrophils and platelets.

\section{Acknowledgments}

This study was partially supported by a grant from the Polish Ministry of Science for Young Scientist (Project No. 01-0221/08/508).

\section{Conflict of interest}

The authors declare no conflict of interest.

\section{References}

1. Huang JT, Abrams M, Tlougan B, et al. Treatment of Staphylococcus aureus colonization in atopic dermatitis decreases disease severity. Pediatrics 2009; 123: 808-18.

2. Alsterholm M, Flytström I, Bergbrant IM, et al. Fusidic acidresistant Staphylococcus aureus in impetigo contagiosa and secondarily infected atopic dermatitis. Acta Derm Venereol 2010; 90: 52-7.

3. Rosińska-Więckowicz A, Czarnecka-Operacz M, Adamski Z. Selected immunological parameters in clinical evaluation of patients with atopic dermatitis. Adv Dermatol Allergol 2016; 33: 211-8.

4. Eliopoulos GM, Thauvin C, Gerson B, et al. In vitro activity and mechanism of action of A21978C1, a novel cyclic lipopeptide antibiotic. Antimicrob Agents Chemother 1985; 27: 357-62.
5. Akins RL, Rybak MJ. In vitro activities of daptomycin, arbekacin, vancomycin, and gentamicin alone and/or in combination against glycopeptide intermediate-resistant Staphylococcus aureus in an infection model. Antimicrob Agents Chemother 2000; 44: 1925-9.

6. Ho SW, Jung D, Calhoun JR, et al. Effect of divalent cations on the structure of the antibiotic daptomycin. Eur Biophy J 2008; 37: 421-33.

7. Julian K, Kosowska-Shick K, Whitener C, et al. Characterization of a daptomycin-nonsusceptible vancomycin-intermediate Staphylococcus aureus strain in a patient with endocarditis. Antimicrob Agents Chemother 2007; 51: 3445-8.

8. Hayden MK, Rezai K, Hayes RA, et al. Development of Daptomycin resistance in vivo in methicillin-resistant Staphylococcus aureus. J Clin Microbiol 2005; 43: 5285-7.

9. Hanifin JM, Rajka G. Diagnostic features of atopic dermatitis. Acta Derm Venereol Suppl (Stockh) 1980; 92: 44-7.

10. Wayne PA. USA: Clinical and Laboratory Standards Institute (CLSI). 2012;M100-S21: Performance standards for antimicrobial susceptibility testing. 21 $1^{\text {st }}$ Informational Supplement.

11. Biomerieux 2008. Etest Daptomycin (DPC) Technical variables that may cause discrepancies in MIC testing. Biomerieux, Inc., CIS 014 (Etest M0000601M10061).

12. Balma-Mena A, Lara-Corrales I, Zeller J, et al. Colonization with community-acquired methicillin-resistant Staphylococcus aureus in children with atopic dermatitis: a cross-sectional study. Int I Dermatol 2011; 50: 682-8.

13. Jappe U, Schnuch A, Uter W. Frequency of sensitization to antimicrobials in patients with atopic eczema compared with non atopic individuals: analysis of multicentre surveillance data, 1995-1999. Br J Dermatol 2011; 149: 87-93.

14. Parra-Ruiz J, Bravo-Molina A, Peńa-Monje A. Activity of linezolid and high-dose daptomycin, alone or in combination, in an in vitro model of Staphylococcus aureus biofilm. J Antimicrob Chemother 2012; 67: 2682-5.

15. Anderl JN, Franklin MJ, Stewart PS. Role of antibiotic penetration limitation in Klebsiella pneumoniae biofilm resistance to ampicillin and ciprofloxacin. Antimicrob Agents Chemother 2000; 44: 1818-24.

16. Barcia-Macay M, Seral C, Mingeot-Leclercq MP, et al. Pharmacodynamic evaluation of the intracellular activities of antibiotics against Staphylococcus aureus in a model of THP-1 macrophages. Antimicrob Agents Chemother 2006; 50: 841-51.

17. Mishra NN, McKinnell J, Yeaman MR, et al. In vitro cross-resistance to daptomycin and host defense cationic antimicrobial peptides in clinical methicillin-resistant Staphylococcus aureus isolates. Antimicrob Agents Chemother 2011; 55: 4012-8.

18. Friedman L, Alder JD, Silverman JA. Genetic changes that correlate with reduced susceptibility to daptomycin in Staphylococcus aureus. Antimicrob Agents Chemother 2006; 50 : 2137-45.

19. Patel JB, Jevitt LA, Hageman J, et al. An association between reduced susceptibility to daptomycin and reduced susceptibility to vancomycin in Staphylococcus aureus. Clin Infect Dis 2006; 42: 1652-3.

20. Sakoulas G, Alder J, Thauvin-Eliopoulos C, et al. Induction of daptomycin heterogeneous susceptibility in Staphylococcus aureus by exposure to vancomycin. Antimicrob Agents Chemother 2006; 50: 1581-5.

21. Mishra NN, Bayer AS, Moise PA, et al. Reduced susceptibility to host-defense cationic peptides and daptomycin coemerge in methicillin-resistant Staphylococcus aureus from daptomycin-naive bacteremic patients. J Infect Dis 2012; 206: 1160-7. 
22. Mishra NN, Yang SJ, Chen L, et al. Emergence of daptomycin resistance in daptomycin-naïve rabbits with methicillinresistant Staphylococcus aureus prosthetic joint infection is associated with resistance to host defense cationic peptides and mprF polymorphisms. PLoS One 2013; 8: e71151.

23. Safdar N, Andes D, Craig WA. In vivo pharmacodynamic activity of daptomycin. Antimicrob Agents Chemother 2004; 48: 63-8.

24. Fuchs PC, Barry AL, Brown SD. In vitro bactericidal activity of daptomycin against staphylococci. J Antimicrob Chemother 2002; 49: 467-70.

25. Johnson AP, Mushtaq S, Warner M. Calcium-supplemented daptomycin Etest strips for susceptibility testing on Iso-Sensitest agar. J Antimicrob Chemother 2004; 53: 860-2.

26. Keel RA, Sutherland CA, Aslanzadeh J, et al. Correlation between vancomycin and daptomycin MIC values for methicillin-susceptible and methicillin-resistant Staphylococcus aureus by 3 testing methodologies. Diagn Microbiol Infect Dis 2010; 68: 326-9.

27. Jevitt LA, Thorne GM, Traczewski MM, et al. Multicenter evaluation of the Etest and disk diffusion methods for differentiating daptomycin-susceptible fromnon-daptomycin-susceptible Staphylococcus aureus isolates. J Clin Microbiol 2006; 44: 3098-104. 